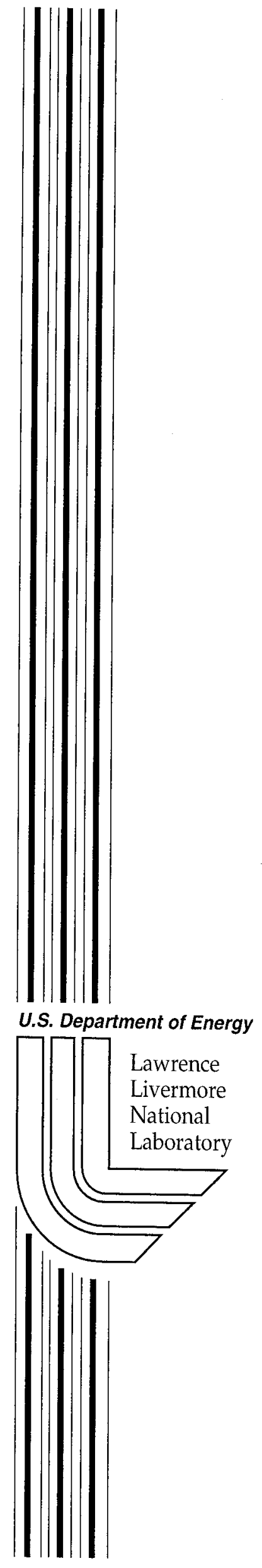

UCRL-ID-138934 Rev 1

\title{
Spheromak Power and Helicity Balance
}

\author{
K. I. Thomassen
}

May 18, 2000 


\section{DISCLAIMER}

This document was prepared as an account of work sponsored by an agency of the United States Government. Neither the United States Government nor the University of California nor any of their employees, makes any warranty, express or implied, or assumes any legal liability or responsibility for the accuracy, completeness, or usefulness of any information, apparatus, product, or process disclosed, or represents that its use would not infringe privately owned rights. Reference herein to any specific commercial product, process, or service by trade name, trademark, manufacturer, or otherwise, does not necessarily constitute or imply its endorsement, recommendation, or favoring by the United States Government or the University of California. The views and opinions of authors expressed herein do not necessarily state or reflect those of the United States Government or the University of California, and shall not be used for advertising or product endorsement purposes.

Work performed under the auspices of the U.S. Department of Energy by the University of California Lawrence Livermore National Laboratory under Contract W-7405-Eng-48.

This report has been reproduced directly from the best available copy.

Available to DOE and DOE contractors from the Office of Scientific and Technical Information P.O. Box 62, Oak Ridge, TN 37831

Prices available from (423) 576-8401 http:// apollo.osti.gov/bridge/

Available to the public from the National Technical Information Service

U.S. Department of Commerce 5285 Port Royal Rd., Springfield, VA 22161 http://www.ntis.gov/

OR

Lawrence Livermore National Laboratory Technical Information Department's Digital Library http://www.llnl.gov/tid/Library.html 


\section{Spheromak Power and Helicity Balance}

This note addresses the division of gun power and helicity between the open line volume and the closed flux surface volume in a steady state flux core spheromak ${ }^{1}$. Our assumptions are that fine scale turbulence maintains each region close to an axisymmetric Taylor state, $\mu_{\mathrm{O}} \mathbf{j}=\lambda \mathbf{B}$.

The gun region that feeds these two volumes surrounded by a flux conserver is shown topologicaly below. (The actual geometry is toroidal). Flux and current from the magnetized gun flow on open lines around the entire closed surface containing the spheromak. The gun current flows down the potential gradient, the potential difference between the two ends of each line being the gun voltage. Here, the gun voltage excludes the sheath drops at each end.

These volumes have different values of $\lambda$ in each region (open line volume $V_{1}$ and closed spheromak volume $V_{2}$ ) and we want to calculate the efficiency of transferring the gun power to the spheromak to sustain the ohmic loss in steady state.

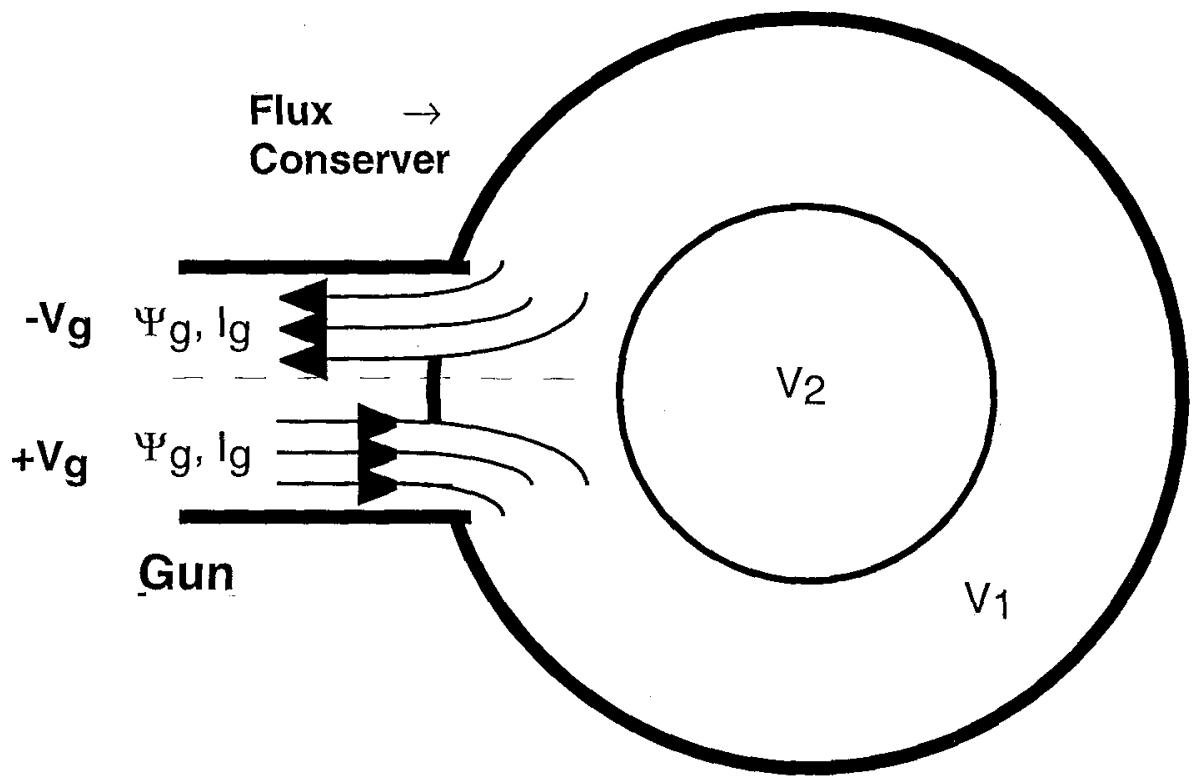

Helicity Conservation

We assume constant $\lambda$-values in each region and a step function drop from region 1 to region 2 , with $\mu_{0} J_{1}=\lambda_{1} B_{1}$ and $\mu_{0} J_{2}=\lambda_{2} B_{2}$. The helicity loss and ohmic powers are;

$$
\frac{\mathrm{dK}}{\mathrm{dt}}=\frac{\mathrm{K}_{1}}{\tau_{1}}=\frac{2 \mu_{\mathrm{o}}}{\lambda_{1}} \mathrm{P}_{\mathrm{oh} 1} \quad \text { and } \quad \frac{\mathrm{dK} 2}{\mathrm{dt}}=\frac{\mathrm{K}_{2}}{\tau_{2}}=\frac{2 \mu_{\mathrm{o}}}{\lambda_{2}} \mathrm{P}_{\mathrm{oh} 2}
$$

with $\tau_{\alpha}=\underset{2 \eta_{\alpha} \lambda_{\alpha}{ }^{2}}{\mu_{0}}$. The gun supplies the total helicity $K_{1}+K_{2}$ to maintain a steady state, 


$$
\frac{d K_{\text {tot }}}{d t}=2 V_{g} \Psi_{g}=\frac{2 \mu_{0}}{\lambda_{1}} P_{\text {gun }}=\frac{K_{1}}{\tau_{1}}+\frac{K_{2}}{\tau_{2}}
$$

so

$$
\mathrm{P}_{\mathrm{g}}=\mathrm{P}_{\text {oh } 1}+\frac{\lambda_{2}}{\lambda_{1}} \mathrm{P}_{\text {oh2 }}
$$

If $f$ region 1 shrinks to zero volume $\left(K_{1}=0\right)$ the ohmic power in region 2 is $\frac{\lambda_{2}}{\lambda_{1}} P_{g}$.

The ratios of ohmic power and gun power are,

$$
\stackrel{\left(P_{\text {oh }}\right) 1}{P_{\text {gun }}}=\left[1+\frac{K_{2} \tau_{1}}{K_{1} \tau_{2}}\right]^{-1} \quad \frac{\left(P_{\text {oh }}\right) 2}{P_{\text {gun }}}=\frac{\lambda_{2}}{\lambda_{1}}\left[1+\frac{K_{1} \tau_{2}}{K_{2} \tau_{1}}\right]^{-1} \quad \text { with } K_{\alpha}=\int \frac{B_{\alpha}^{2}}{\lambda_{\alpha}} d V_{\alpha}
$$

Power Balance

Now let us balance the gun power with losses inside the closed volume. In what follows we assume there is a non-zero mean value of the products $E \cdot B$ and j•E which determine helicity loss and power loss respectively. First, the flow of power into region 1 is along the open lines, where there is an electric field consisting of the dynamo field $\mathbf{E}_{\text {dyn1 }}$ (which may or may not time average to zero) and the ohmic field $\eta_{1} j_{1}$. There is a flow of power across the separatrix surface which we call $P_{2}$. So we can equate the inward flow of power from the gun to loss of power in the volume plus flow of power out of region 1 into region 2;

$$
P_{g}=\int j_{1} \cdot E_{1} d V_{1}+P_{2}=\int j_{1} \bullet E_{d y n 1} d V_{1}+\int \eta_{1} j_{1}{ }^{2} d V_{1}+P_{2}
$$

That power flow into region 2 sustains the field against ohmic losses that would otherwise cause the stored energy to decay and feeds the dynamo in that region.

$$
P_{2}=\int j_{2} \cdot E_{d y n 2} d V_{2}+\int \eta_{2} j_{2}{ }^{2} d V_{2}
$$

Adding these and using the fractions of gun power going to ohmic heating,

$$
P_{g}-P_{\text {oh1 }}-P_{\text {oh2 }}=\int j \cdot E_{d y n} d V=\left(\frac{\lambda_{1}}{\lambda_{2}}-1\right) P_{\text {oh2 }}=P_{\text {dyn }}
$$

The dynamo term is non-zero only where there is a gradient in $\lambda$, as we will see later, and as $\lambda_{1}$ approaches $\lambda_{2}$ the need for a dynamo vanishes and that power is zero (a state one cannot actually reach). The dynamo power first goes into waves or MHD modes but eventually into the plasma ions and/or electrons according to details of the processes that try to maintain a Taylor state.

The gun power provides the input for dynamo power and ohmic heat, but each of these three $\left(P_{\text {dyn }}, P_{\text {oh1 }}\right.$, and $P_{\text {oh2 }}$ ) can also be viewed as plasma input power, and we could write diffferent equations that distribute this input heat and 
wave power to various loss channels, such as radiation, power to restore charge exchange ion losses, conduction or convection loss, etc. To understand how the dynamo power is distributed one needs more detail on the process, which we will not present in this note.

\section{Dynamo Transport}

We assumed so far that the $\lambda$-values were constant in each region, with an infinite gradient at the boundary of the two regions. In reality there is some gradient everywhere, and integrals involving $\mathrm{j}$ use $\mu_{\mathrm{o}}{ }^{-1} \lambda \mathrm{B}$ so that $\lambda$ cannot be taken outside of those integrals as we have done. To understand better the role of the $\lambda$-gradients let us use a model suggested by Hooper ${ }^{2}$ from the work of Boozer ${ }^{3}$ and Strauss ${ }^{4}$. The model gives the mean of a product of the dynamo electric field and magnetic field, valid for small-amplitude fluctuations, containing the $\lambda$-gradient and $\kappa, a$ hyper-resistivity,

$$
\mathbf{E}_{\mathrm{dyn}} \cdot \mathbf{B}=-\nabla \cdot\left\{\frac{\kappa \mathrm{B}^{2}}{\mu_{\mathrm{o}}} \nabla \lambda\right\}
$$

Then, a calculation of the dynamo power loss in a volume $V$ is

$$
\begin{aligned}
\int j \bullet E_{d y n} d V & =\int \frac{\lambda}{\mu_{0}} B \cdot E_{d y n} d V=-\int \frac{\lambda}{\mu_{0}} \nabla \cdot\left\{\frac{\kappa B^{2}}{\mu_{0}} \nabla \lambda\right\} d V \\
& =-\int \nabla \cdot \frac{\lambda}{\mu_{0}}\left\{\frac{\kappa B^{2}}{\mu_{0}} \nabla \lambda\right\} d V+\int\left\{\frac{\kappa B^{2}}{\mu_{0}} \nabla \lambda\right\} \bullet \frac{\nabla \lambda}{\mu_{0}} d V
\end{aligned}
$$

If we first apply this result to the entire volume inside the flux conserver, the first integral can be converted to a surface term which is zero on the walls since $\nabla \lambda$ is zero there. There are two wall surfaces, one where the gun flux enters the volume and the remainder where the flux is parallel to the wall. In either region, $\nabla \lambda$ is zero at the wall. The remaining term can be written $\int \mathrm{kj}^{2} \frac{[\nabla \lambda]^{2}}{\lambda^{2}} \mathrm{dV}$, which suggests that the strength of the dynamo in a given spot is inversely proportional to the square of the gradient length there, and that there is no dissipation of dynamo power without a gradient in $\lambda$.

We now integrate $\mathbf{j} \cdot \boldsymbol{E}_{\mathrm{dyn}}$ in volume 1 , where there are two kinds of surfaces, the flux conserver and the separatrix surface between regions. The surface integral is not zero on the latter, so that our power $\mathrm{P}_{2}$ that flows into region 2 is proportional to $\nabla \lambda$ on that surface. With $S_{1}$ the common surface connecting the two regions,

$$
\mathrm{P}_{2}=\oint \frac{\lambda}{\mu_{\mathrm{o}}}\left\{\frac{\kappa \mathrm{B}^{2}}{\mu_{\mathrm{o}}} \nabla \lambda\right\} \mathrm{dS} \mathrm{S}_{1} \quad \text { and } \quad \mathrm{P}_{\mathrm{dyn} 1}=\int \kappa j_{1}{ }^{2} \frac{[\nabla \lambda]^{2}}{\lambda^{2}} \mathrm{dV} \mathrm{V}_{1}
$$


So, the dynamo in region 1 supplies some power to region 2 but some is dissipated if there is a $\lambda$-gradient in region 1 . Integrating $j \cdot E$ in region 2 gives three terms, the dynamo surface term above, any dynamo volume dissipation if $\nabla \lambda \neq 0$ inside the volume, and $P_{\text {oh2 }}$.

If we assume the $\lambda$-values are constant in each region, with a jump in $\lambda$ at the bounding surface, then all the helicity flow and power dissipation from the excess $\lambda_{1}$ $>\lambda_{2}$ would take place there. In reality, as we indicated here, it is distributed. When it is, the simple calculations above must take into account the spatial changes in $\lambda$.

These results are presented only to qualitatively understand power density and flow as they relate to $\lambda$-gradients. The concept of hyper-resistivity may not be fully applicable here. And, we point out the obvious, that the calculations of this note are for axisymmetric ideal spheromaks kept at the Taylor state by fine grain turbulence. The calculations are done for the purpose of better understanding the gun power balance, not for understanding the physics of spheromaks driven by large amplitude low mode number (both axisymmetric and non-axisymmetric) instabilities.

\section{Power and Helicity Loss}

In this section the fraction of ohmic power and helicity to the open and closed field regions during steady state is given based on a simple model below of a gun driven spheromak. The fields in the closed line region 2 are modeled by those of a closed cylinder of height $L$, radius $R$. The open lines in region 1 are in a column of radius $R_{1}$ on the geometric axis and a return annulus with inner and outer radii of $R_{2}$ and R. From this model we generated Table 1, showing the fraction of stored energy in regions 1 and 2, normalized to the stored energy if $R_{1} \rightarrow 0$. From these results we compute helicities as a percent of the total and ohmic power as a fraction of the gun power. It is assumed that the $\lambda$-gradient is all on the surface between the regions.
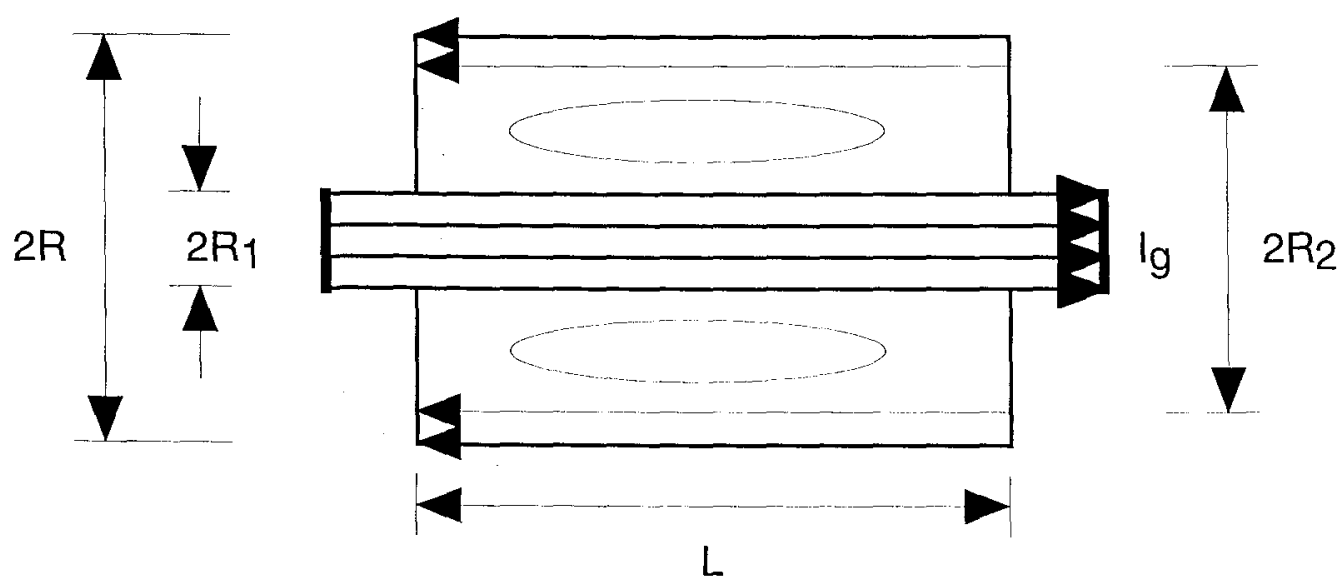

The fields inside the closed flux region are given by 
$\mathrm{B}_{\mathrm{r} 2}=\frac{\mathrm{k}}{\lambda_{2}} \mathrm{~B}_{\mathrm{O} 2} \sin (\mathrm{kz}) \mathrm{J}_{1}(\mu \mathrm{r}) \quad \mathrm{B}_{\phi 2}=\mathrm{B}_{\mathrm{0} 2} \cos (\mathrm{kz}) \mathrm{J}_{1}(\mu \mathrm{r}) \quad \mathrm{B}_{\mathrm{z} 2}=\frac{\mu_{1}}{\lambda_{2}} \mathrm{~B}_{\mathrm{o} 2} \cos (\mathrm{kz}) \mathrm{J}_{\mathrm{O}}(\mu \mathrm{r})$ with $\mathrm{kL}=\pi, \mu \mathrm{R}=3.83, \lambda_{2}{ }^{2}=\mathrm{k}^{2}+\mu^{2}$.

In the open line region the assumption that the lines are straight is valid when $L>R$, and we will assume the radial extent is sufficiently small that both the radial and toroidal fields can be neglected in these columns (we will be integrating $\mathrm{B}^{2}$ for helicity). Across the boundary of the two regions we must increase the ratio of current to field, so we match the $B_{z}$ fields and make a jump in the current $j_{z}$ and the (very small) toroidal field to account for the higher $\lambda$. The fields in region 1 are then $B_{r 1, \phi 1} \approx 0$ and $\lambda_{1} B_{z 1}=\mu B_{01} \cos (k z) J_{0}(\mu r)$. There is a scale factor change in $B_{0}$ by the $\lambda$-ratio, $\lambda_{1} B_{02}=\lambda_{2} B_{01}$, for continuity of $B_{Z}$ into region 2 .

We want to calculate the helicities $\lambda_{\alpha} K_{\alpha}=\int B_{\alpha}{ }^{2} d V_{\alpha}=2 \mu_{0} W_{\text {mag }}=2 \mu_{o}\left(P_{o h}\right)_{\alpha} \tau_{\alpha}$ where the time constant $\tau_{\alpha}$ is $2 \eta_{\alpha} \lambda_{\alpha}{ }^{2} \tau_{\alpha}=\mu_{0}$. In region 2 , with $2 \mu_{0} W_{0}=\pi R^{2} L_{02}{ }^{2}$, and with $r=x R, x_{1} R=R_{1}, x_{2} R=R_{2}$. Then,

$$
\underset{W_{0}}{W_{\text {mag2 }}}=\int_{x_{1}}^{x_{2}}\left\{1+\left(\frac{k}{\lambda_{2}}\right)^{2} J_{1}^{2}(3.832 x)+\left(\frac{\mu}{\lambda_{2}}\right)^{2} J_{0}^{2}(3.832 x)\right\} x d x
$$

For $\mathrm{x}_{1}=0$ this integral is $\mathrm{c}^{2}=\mathrm{J}_{0}^{2}(3.832)=0.162$. In region 1 ,

$$
W_{\text {mag1 }}^{W_{0}}=\left(\frac{\mu}{\lambda_{2}}\right)^{2}\left\{\int_{0}^{x_{1}} x J_{0}^{2}(3.83 x) d x+\int_{x_{2}}^{1} x J_{0}^{2}(3.83 x) d x\right\}
$$

The normalized radius $x_{2}$ is found from $x_{1}$ by returning all the column flux into the outside annulus, $\Phi=\int_{0}^{R_{1}} 2 \pi r B_{z 2}(r, 0) d r=\int_{R_{2}}^{R} 2 \pi r B_{z 2}(r, 0) d r$. But at the outer edge $B_{z}$ is $c$ $=-40 \%$ of what it is on the $r=0$ axis so for small $x_{1}$ the radius $R_{2}$ is found from $R_{1}{ }^{2}=$ $0.4027\left[R^{2}-R_{2}^{2}\right]$ and $x_{2}^{2}=1-2.483 x_{1}{ }^{2}$. If we further assume $L=R$, as in SSPX, so that $\left(\frac{\mu}{\lambda_{2}}\right)^{2}=0.6$ and $\left(\frac{k}{\lambda_{2}}\right)^{2}=0.4$ we get the results in Table 1 below.

Results

The stored energies in the Table are given as a percent of $c^{2} W_{0}$, and the helicities are a percent of the total. Ohmic power loss is a percent $\left(p_{1,2}\right)$ of the gun power, and the power balance formulas from above are,

$$
\underset{P_{\text {oh1 }}}{\mathrm{P}_{\mathrm{g}}}=1+\frac{\eta_{2} \lambda_{2} W_{2}}{\eta_{1} \lambda_{1} W_{1}} \quad \underset{\mathrm{P}_{\mathrm{oh} 2}}{\mathrm{P}_{\mathrm{g}}}=\frac{\lambda_{1}}{\lambda_{2}}\left(1+\frac{\eta_{1} \lambda_{1} W_{1}}{\eta_{2} \lambda_{2} W_{2}}\right) \quad P_{\text {dyn }}=\left(\frac{\lambda_{1}}{\lambda_{2}}-1\right) P_{\text {oh2 }}
$$


From $P_{\text {oh2 }}=\frac{W_{2}}{\tau_{2}}$ we get the fraction of the gun power sustaining the spheromak. The helicity percentages (of the total) in regions 1 and 2 are $h_{1,2}$

$$
\left(h_{1}\right)^{-1}=1+\frac{\lambda_{1}}{\lambda_{2}} \frac{W_{2}}{W_{1}} \quad\left(h_{2}\right)^{-1}=1+\frac{\lambda_{2}}{\lambda_{1}} \frac{W_{1}}{W_{2}}
$$

In the table, $\mathrm{n}=\frac{\eta_{1}}{\eta_{2}}$ and $\mathrm{L}=\frac{\lambda_{1}}{\lambda_{2}}$ from which we compute $T=\frac{\tau_{2}}{\tau_{1}}$.

The primary result is quite evident. When the open line volume is small, but its resistivity is high relative to that in the spheromak, most of the gun power available for increasing stored energy (through sustaining and exceeding the ohmic loss) goes to the open lines. Further, the difference of $p_{1}$ and $p_{2}$ from $100 \%$ is the power available for the dynamo. In all these cases, for $\lambda_{1}=\lambda_{2}$, the dynamo power is equal to $P_{\text {oh2 }}$. For the higher resistivity ratios it is reduced, since it must feed the helicity decay in region 1. Although that helicity is small, its rate of decay is very large. Of course, in the limit of a very hot spheromak nearly all the gun power sustains the edge.

\section{Table 1}

Helicity and power fractions as functions of $\eta, \lambda$

$\begin{array}{cccccccccccc}\text { x1 } & \mathrm{x} 2 & \% \mathrm{Vol} & \mathrm{n} & \mathrm{L} & \mathrm{T} & \% \mathrm{w} 1 & \% \mathrm{w} 2 & \mathrm{~h} 1 & \mathrm{~h} 2 & \mathrm{p} 1 & \mathrm{p} 2 \\ 0.05 & 0.997 & 0.871 & 5 & 2 & 20 & 0.272 & 99.72 & 0.136 & 99.86 & 2.66 & 48.67 \\ 0.1 & 0.988 & 3.483 & 5 & 2 & 20 & 1.038 & 98.89 & 0.522 & 99.48 & 9.5 & 45.25 \\ 0.14 & 0.975 & 6.827 & 5 & 2 & 20 & 1.917 & 97.8 & 0.971 & 99.03 & 16.4 & 41.81 \\ 0.18 & 0.959 & 11.28 & 5 & 2 & 20 & 2.925 & 96.34 & 1.495 & 98.5 & 23.3 & 38.35 \\ & & & & & & & & & & & \\ 0.05 & 0.997 & 0.871 & 10 & 2 & 40 & 0.272 & 99.72 & 0.136 & 99.86 & 5.17 & 47.41 \\ 0.1 & 0.988 & 3.483 & 10 & 2 & 40 & 1.038 & 98.89 & 0.522 & 99.48 & 17.4 & 41.32 \\ 0.14 & 0.975 & 6.827 & 10 & 2 & 40 & 1.917 & 97.8 & 0.971 & 99.03 & 28.2 & 35.92 \\ 0.18 & 0.959 & 11.28 & 10 & 2 & 40 & 2.925 & 96.34 & 1.495 & 98.5 & 37.8 & 31.11 \\ & & & & & & & & & & & \\ 0.05 & 0.997 & 0.871 & 50 & 2 & 200 & 0.272 & 99.72 & 0.136 & 99.86 & 21.4 & 39.28 \\ 0.1 & 0.988 & 3.483 & 50 & 2 & 200 & 1.038 & 98.89 & 0.522 & 99.48 & 51.2 & 24.39 \\ 0.14 & 0.975 & 6.827 & 50 & 2 & 200 & 1.917 & 97.8 & 0.971 & 99.03 & 66.2 & 16.89 \\ 0.18 & 0.959 & 11.28 & 50 & 2 & 200 & 2.925 & 96.34 & 1.495 & 98.5 & 75.2 & 12.39 \\ 0.2 & 0.949 & 13.93 & 50 & 2 & 200 & 3.443 & 95.49 & 1.771 & 98.23 & 78.3 & 10.86\end{array}$

The current $I_{g}=j_{1} x_{1} 2\left[\pi R^{2}\right]$ flows through a resistance $\eta_{1}$ (length/area). The length of both the column and the return annulus is $L=R$, while the normalized areas are $x_{1}{ }^{2}$ and $1-x_{2}{ }^{2}$. So the resistance is $\frac{1.4 \eta 1}{x_{1}^{2} \pi L}=\frac{P_{\text {oh1 }}}{I_{g}^{2}}=p_{1}(\%) \frac{V_{g}}{l_{g}}$. 
References

1. J. B. Taylor, "Relaxation and magnetic reconnection in plasmas," 1986, Rev. Modern Physics, 58, 741

2. E. B. Hooper, SSPX technical note - in progress

3. A. H. Boozer, "Ohm's law for mean magnetic fields," 1986, J. Plasma Phys. 35 , 133

4. H. R. Strauss, 1985 "The dynamo effect in fusion plasmas,", Phys. Fluids 28, 2786

Appendix

We've used the relationship between power and helicity rate for regions of constant $\lambda, P_{\text {oh }}=\frac{\lambda}{2 \mu_{0}} \frac{d K}{d t}$ dependent on the guage invariant form of helicity,

$$
K_{0}=\int A \cdot B d V=\int \lambda^{-1} B \cdot B d V=\lambda^{-1}\left(2 \mu_{0} W_{\text {mag }}\right) \quad(\lambda=\text { constant })
$$

In steady state the loss of helicity and the rate at which magnetic energy is converted to ohmic losses can be equated, $\frac{\lambda}{2 \mu_{0}} \frac{d K_{0}}{d t}=\stackrel{d W_{\text {mag }}}{d t}=P_{\text {oh. }}$.

Although the separatrix boundary between regions 1 and 2 is not a conductor, we assume that the mean magnetic fields lie in flux surfaces so that B•n $=0$ on that surface. The regions are simply connected and the helicity $\mathrm{K}_{\mathrm{O}}$ is gauge invariant, and helicity can be defined in each region.

Nonetheless, we could use another form for helicity which is always gauge invariant ${ }^{1}, K=K_{0}-\oint A \bullet d \ell_{p} \oint A \bullet d \ell_{T}$. Here, the first closed path integral is the short way (poloidal) around a flux surface boundary $\left(B_{n}=0\right)$, and the second is the long way around (toroidal). Using the separatrix boundary surface to evaluate them, the toroidal flux inside the separatrix (region 2) is $\Phi_{T}=\lambda \oint A \bullet d \ell_{p}$. Also, $\lambda \oint A \bullet d \ell_{T}=$ $\oint B \bullet d l_{T}=2 \pi R B_{T}$ (note that $R B_{T}$ is constant, so the toroidal path can be taken anywhere on the flux surface). Now, though $\lambda \mathrm{K}$ and $2 \mu_{0} W_{m}$ are related differently than is $\lambda K_{0}$, our integrals are constant during equilibrium so $\frac{d K}{d t}$ can be related directly to ohmic power.

Acknowledgement

This work was performed under the auspices of the U.S. Department of Energy by the University of California, Lawrence Livermore National Laboratory, under contract No. W-7405-Eng-48. 